\title{
Comparison of Non-Contact ACL Injury vs. No Injury in Female Identical Twin Athletes
}

\author{
Matthew Nowland $^{1 *}$, Karen Myrick ${ }^{1,2}$, Lynn Copes $^{1}$, Juan C. Garbalosa ${ }^{3}$, Richard Feinn ${ }^{1}$ \\ ${ }^{1}$ Frank Netter School of Medicine, Quinnipiac University, Connecticut, USA \\ ${ }^{2}$ University of Saint Joseph, School of Interdisciplinary Health and Science, Connecticut, USA \\ ${ }^{3}$ Quinnipiac University, School of Health Sciences, Connecticut, USA
}

Received Date: April 02, 2020; Accepted Date: April 09, 2020; Published Date: April 17, 2020

*Corresponding Author: Matthew Nowland, Frank Netter School of Medicine, Quinnipiac University, 161 Westminster Street, Hamden, CT 06518, USA. Tel: +978866-6414; Email: Matthew.Nowland@ Quinnipiac.edu

\section{Abstract}

Background: Anterior cruciate ligament injuries are associated with significant morbidity. A variety of tests and criteria have been proposed to identify athletes at risk for reinjury and determine their readiness to return to sport. Biomechanical function of the ACL is an independent predictor of future failure. Genetics and environmental factors play significant roles in ACL injuries. Attempting to try to better understand their influence could have a positive impact on how ACL injuries could be prevented or treated.

Purpose: This project examined a pair of identical twins, one of whom sustained an ACL injury and the other did not. By examining identical twins, the genetic component would be controlled to help determine: 1. If any of the measures of biomechanics may be associated with an ACL injury. 2. If no biomechanical difference, is there an association in the athletic training that potentially increases risk for an ACL injury.

Study Design: 28 D1 female athletes completed a series of agility tests while their trunk and lower extremity biomechanics were recorded. The data obtained was used to assess the biomechanical profiles of the athletes.

Methods: We obtained data from the twins and compared it to the other athletes. We interviewed the twins to compare differences in their lifestyle.
Results: The biomechanical profiles showed no statistical difference. They were consistent with the baseline from the other athletes. In the interviews, the twins were similar in lifestyle except they played different positions in lacrosse.

Conclusion: Based upon the return to sport criteria, the twin who suffered the ACL injury has achieved the criteria. She had successfully return to the same biomechanical profile as the baseline. No factors could be predicted.

Keywords: Identical Twins; Non-contact ACL Injury; Readiness to Return to Sport; Sports Injuries

\section{Introduction}

The anterior cruciate ligament (ACL) injury is the most common musculoskeletal injury in orthopedic sports medicine in the United States. There is roughly a quarter of a million ACL injuries in a year and close to 100,000 ACL repairs [1-3]. The greatest percentage of individuals who suffer an ACL injury are younger athletes ages 15-25 years of age [1, 4]. Even though the ACL injury is relatively common, along with repair, the timeframe of recovering is significant, requiring extensive therapy and training $[\mathbf{5}, \mathbf{6}]$. This takes away from time playing a sport or performing many athletic activities and can have a major toll on an individual's psychological confidence and well-being. In terms of longitudinal cost, ACL repairs are both financially costly for the initial repair and the consequences in the future. ACL injuries predispose individuals to

1 | Advances in Orthopaedics and Sports Medicine, Volume 2020, Issue 01 
increased risk for secondary injuries, such as tearing any repaired ligament, meniscal tears, and injuring other ligaments in the knee. Furthermore, ACL injuries increase the risk of developing osteoarthritis $[\mathbf{5 , 7 - 8}]$. The total financial burden for an individual across their lifetime is estimated to be between $\$ 38,121$ to $\$ 88,538$, depending on the surgical repair or treatment of the injury. This is estimated to be a total cost in the United States of over 2 billion dollars [3]. Between the cost, frequency, and lifetime burden an ACL injury has on an individual and the healthcare system, it is essential to be able to research better evidence-based ways to prevent and treat ACL injuries that are more cost and time effective.

There are quite a few risk factors for an ACL injury that have been the subject of extensive research [2,9-14]. Many of these risk factors, as one might expect in a sport injury, are modifiable. Those specifically have been a primary focus in hoping to be able to find better ways to decrease the incidence of ACL injuries. Much of the research has been dedicated to preventative programs focused on the modifiable risk factors. The majority of the modifiable risk factors are based upon the individual or an athlete maneuvers during landing, deceleration, or changing of direction movements [15-18]. The programs have found success at training individuals in better techniques to prevent injuries, but even so the overall incidence rate of an ACL injury is still quite high in the United States. There is also a discrepancy between the incidences in male athletes compared with female athletes, with females having a higher incidence even though the cause is the same [17-20]. With the incidence continuing to be high, further research is needed to re-examine the modifiable risk factors as well as try to identify other potential risk factors that can be addressed. This raises the question of whether the most important factors leading to an ACL injury have been truly identified or not.

In the case of an ACL injury and the rehabilitation from the repair, many individuals undergo some type of program with a goal of returning to their previous degree of activity. In regards to athletes, this is referred to a return to sport criteria. Unfortunately, there are not any concrete guidelines on a return to sport criteria and it can vary dramatically between rehabilitation programs [21]. For some programs, their criteria are the length of time from the surgical repair. Other Programs may have their criteria based upon the difference between the strength of the muscle in the repaired leg compared to the uninjured leg, or the size difference between their thighs. Lastly, some programs used criteria based upon physical fitness tests to determine if the individual could perform the same physical tasks that would have been expected prior to surgery on each leg.

One aspect lacking in the research of risk factors for sustaining an ACL injury is an understanding of genetic factors that may contribute to individual variation in ACL strength or elasticity, locomotor behaviors, and/or response to pre- or post-rupture training. The opportunity to examine identical twins, only one of whom has suffered a ruptured ACL is novel. Because the subjects are identical twins, their genetic makeup will be essentially identical, providing a large degree of control over genetic differences between the two athletes.

As part of their preseason training program, 28 female D1 lacrosse athletes completed a battery of agility tests. All subjects completed a brief questionnaire about their past injury history, treatment for these injuries, and age. Among the athletes who participated were identical female twins. One of the twins had previously sustained a non-contact ACL rupture and the other had not. Prior to conducting an interview and analyzing their biomechanical profiles, informed consent was obtained from each subject. The objective of this report is to:

1. Determine if there are any biomechanical differences between the subjects and if these differences could make an athlete more susceptible to an ACL injury.

2. Determine if there are any differences in the ways the twins trained or competed that may predict whether or not an athlete may be more susceptible to an ACL injury.

3. To examine the return to sport criteria with the twin who underwent the ACL repair and see if they had undergone a post-surgery rehabilitation program that allowed them to achieve the same degree of physical fitness level as their identical twin.

4. Specifically, we are examining the return to sport criteria in the twin with the non-contact ACL injury. We are examining them to see if they had achieved both a return to sport in physical play and achieved biomechanical profile similar to an individual without a previous ACL injury. This will be done by comparing the twin to both her identical twin who did not have an injury and a baseline cohort of the rest of the athletes.

We hypothesized that the subjects would exhibit differences in their biomechanical and their training profiles.

\section{Materials and Methods}

\section{Previous Data}

As part of their preseason training program, 28 female D1 athletes completed a battery of agility tests while retro reflective markers placed on their trunks and lower extremities were recorded using a 16-camera motion analysis system. All subjects completed a brief questionnaire about their past injury history, treatment for these injuries, and age. The agility tests consisted of one 90-minute data collection session and included a series of jump and cutting tests.

After a warm-up period consisting of running on a treadmill and lower extremity stretches, retro reflective markers were placed to the skin of the athletes. The markers were put on specific bony landmarks located on the subjects' lower and upper extremities, trunk, and pelvis. A 16-camera motion capture system was used to initially collect a 6 second trial of video data at a sampling rate of $300 \mathrm{~Hz}$ with the athletes standing in the middle of the motion capture with their hips and knees in 0 degrees of flexion. The athletes then proceeded to perform specific physical exercises to assess their 
biomechanical profile. These tests were hop and agility tests and a weighted squat test, the order of which was randomized. Each athlete was given three opportunities for each exercise with a 30 second rest between the repetitions and a 1 minute rest between the exercises. The exercise was each demonstrated to the athlete prior to the athlete performing the exercise. The three tests were: a hop tests consisting of a cross over single leg jump for distance performed bilaterally, an agility tests consisting of a modified T-test, a straight forward and backward run, and a shuffle test, and a weighted squat test consisting of a squat with an Olympic barbell for 5 repetitions. During the exercises, kinematic and kinetic data was collected at the same time with the 16-camera motion capture system. All participants were free of injury at the time of participation, cleared to participate in games and practice by their health care provider, and did not have any musculoskeletal, cardiovascular, or neuromuscular condition that was contraindicated for participation.

\section{Statistical Analysis}

Using linear mixed models a comparison of means between each of the twins and also between the twins and the other athletes was conducted. Fixed effects included twin and agility test and random effects included the intercept for repeated observations within in the same agility test and agility tests within in the same athlete. Tests were carried out for each of the three agility tests on both the knee and hip during initial contact and flexion.

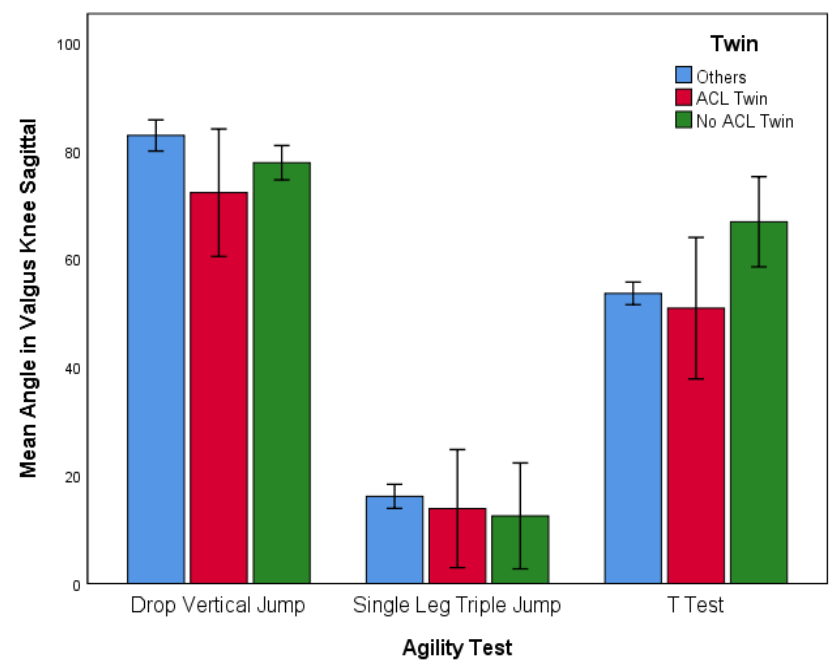

(a)
Informed Consent and Institutional Review Board (IRB): Informed consent from each of the twins was obtained prior to conducting the interview and beginning the case report. An IRB was obtained for a case report on examining the twins biomechanical function, comparing it to the cohort, and interviewing the twins.

\section{Interview}

Each twin was interviewed using a questionnaire of the same seven questions. The questions were based on sport played, their role in the sport, training, exercise, physical hobbies, and diet. The twin with the non-contact ACL injury also had an additional question on the type of ACL reconstruction performed.

\section{Results}

\section{Statistical Analysis}

The data analysis showed that each of the twins compared to each other had a similar biomechanical profile during their agility tests (all p-values >.05). The data showed that for both their hip and knee profile, they were relatively the same in all planes. Furthermore, both of the twin's biomechanical profiles did not differ from the baseline cohort of the other 26 individuals (all p-values $>.05$ ).

In (Figure 1a and 1b) both twins demonstrate that their knees were of similar angular movement in the sagittal plane during valgus and flexion.

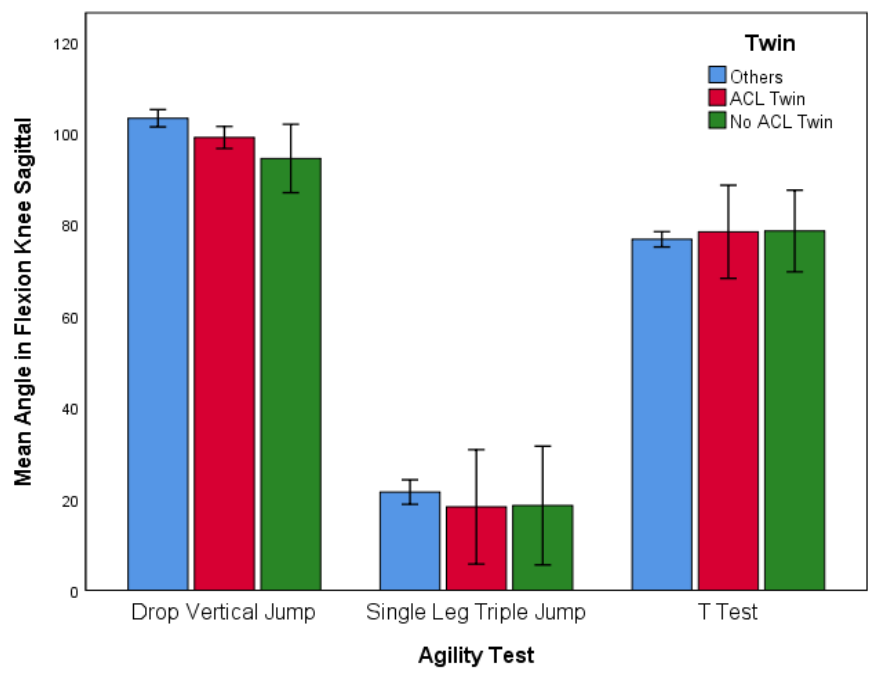

(b)

Figure 1a, 1b: A sagittal view snapshot of the knee during the agility tests of a drop vertical jump, single leg triple jump, and T test respectively.

In (Figure 2a and 2b), the athlete's knees were viewed during valgus and flexion as they performed the agility tests. Both twins demonstrate that their biomechanical profile were similar to the rest of the athlete's. 


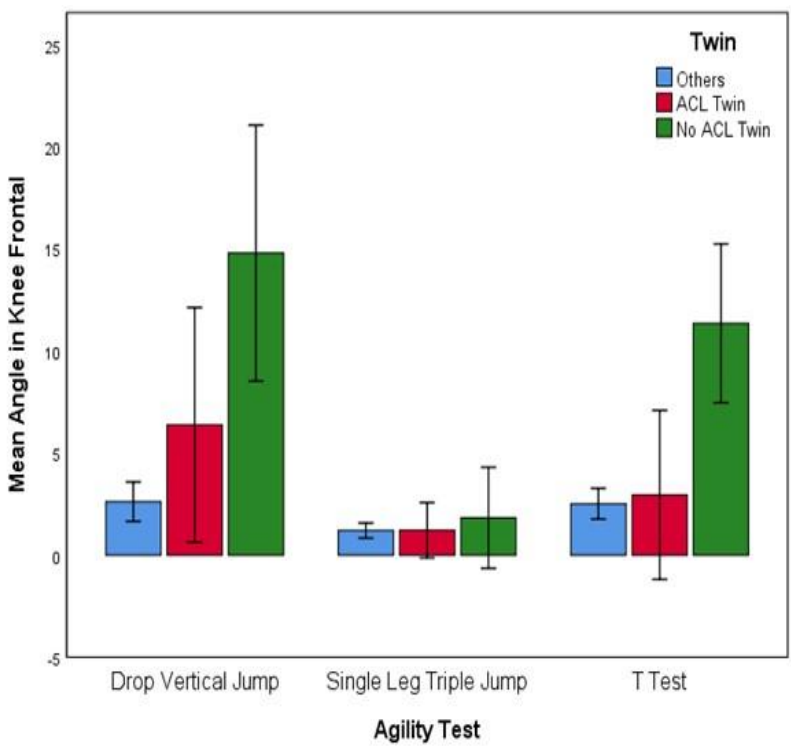

(a)

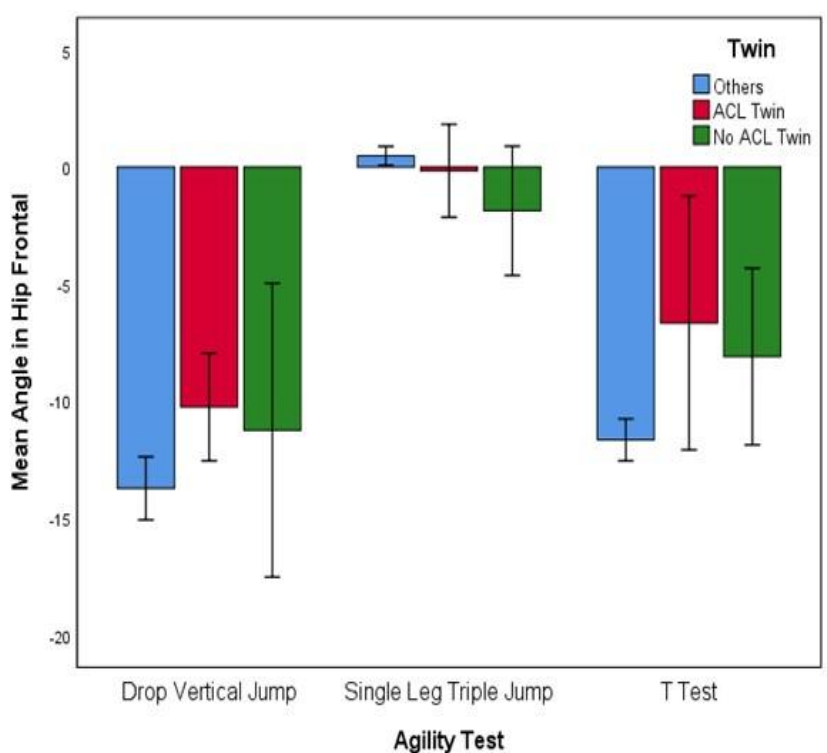

(b)

Figure 2a, 2b: A frontal view snapshot of the knee and hip in flexion during agility tests of a drop vertical jump, single leg triple jump, and $\mathrm{T}$ test respectively.

In (Figure 3), the athlete's movement of their knee into flexion was measured based upon length of time. Both twins during the agility tests had similar results as the rest of the athletes in length of time to reach maximum knee flexion of their knee.

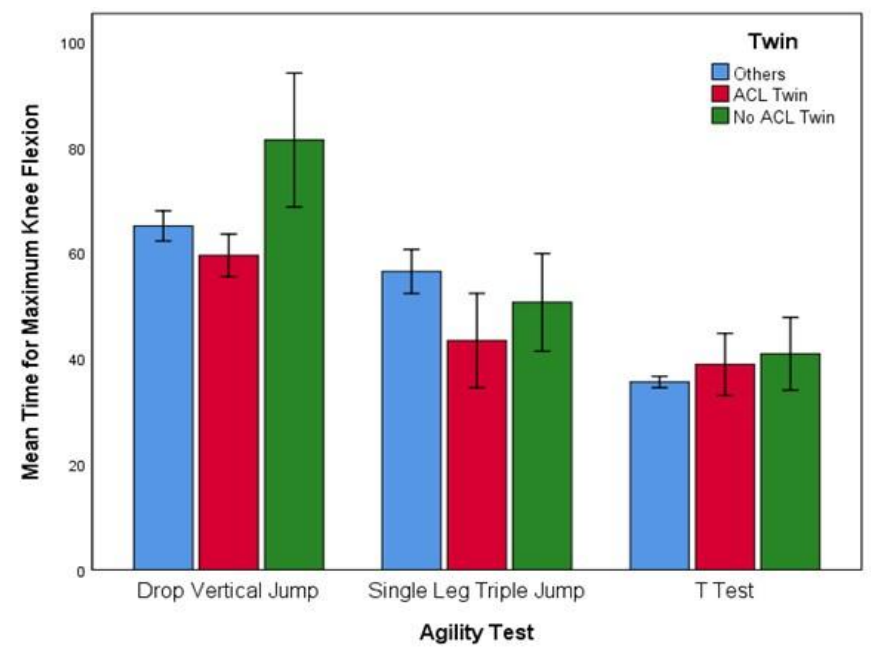

Figure 3: Biomechanical range for the knee during flexion taken over a length of time during the agility tests of a drop vertical jump, single leg triple jump, and $\mathrm{T}$ test respectively.

\section{Interview}

Based upon the questionnaire, both the twin athletes led very similar lives. Most of their life was virtually the same as they did everything together. They both followed the same gym routine. They both followed the same hobbies: biking, hiking, and volleyball. They both followed a higher protein, low carb diet consisting of three servings of fruit a day, three servings of vegetables a day, and two servings of dairy a day.
Outside of their respected sport, the only major lifestyle difference between the twins was the twin with the previous ACL injury also surfed. She picked up surfing after having the ACL repair to help retrain her balance. Where they differed was that each twin played a different position in their respected sport of lacrosse. The twin without the previous injury played the forward position in lacrosse. She claims that this position is a purely offensive position and consists of short distance running with a lot of dodging and changing 
direction. This is a very fast pace play style. The training consists of working in a lot of tight spaces to create change rapid change of direction and speed. The twin with previous non-contact ACL injury played midfield position in lacrosse. She claims that this position requires the same training as the forward position, but also additional training for defense. Defensive play style is more reactive, running in more linear fashion, and standing in a lower stance. Their training consists of everything the offensive positions train in, and also incorporating more ladder work, footwork drills, and shuffling side to side.

The twin with the non-contact ACL injury underwent a routine ACL reconstruction and return to play rehabilitation program. The type of ACL repair the athlete elected to have was a patella ligament repair. The surgery was followed by a return to sport physical therapy with three hour sessions three times a week for six months.

\section{Discussion}

An ACL tear is a very common sports injury that requires surgery and a lengthy period of rehabilitation in order to return to the athlete's previous level of play. The required task to successfully be able to return to a sport can be daunting, and many athletes run into secondary injuries after their ACL repair [7]. Our hope was that by looking at the biomechanical profile of the hip and knee during agility tests in two identical twins, where one had a non-contact ACL injury and the other did not, we would be able to evaluate the return to sport criteria.

By specifically examining identical twins we were able to reduce any genetic difference between the individuals to a minimum. This allowed us to better evaluate whether the twin with the ACL repair had achieved the ability to return to sport after undergoing a rehabilitation program in comparison to their identical twin. Since the twin who had the ACL repair was playing in their respected sport, we questioned if she would perform with a similar biomechanical profile as the baseline her twin. We assumed that the average of all the other athletes would form a baseline that would also be consistent with the twin who had never had an injury. Even though it was unknown if any of the other athletes had a previous injury and also gone through a rehabilitation program, that uncertainty was not important. Due to the sample size, we assumed that the average of all the students would be most similar to a baseline of an individual who had never had a previous injury, like one of the twins. The question therefore, was whether the twin with the previous non-contact ACL injury would have had a successful previous rehabilitation program, following a return to sports criteria that allowed her to have a biomechanical profile that was similar to the baseline of the other athlete's.

All the data showed that the twin with the non-contact ACL injury did in fact have a biomechanical profile with the same baseline as the rest of the athletes. Although this does not help identify any areas that could possibly be improved, it does show that the steps taken by the twin allowed her to fulfill the physical requirements to reach the return to sport criteria. Based upon the interview, the twin had to undergo a rigorous rehabilitation program with extensive physical therapy. Likely due to the physical therapy and the rehabilitation training she received, when doing the agility tests, she had a biomechanical profile similar to both her twin and the rest of the athletes. It is interesting to note the differences between the twins in terms of athletic play. The twin that did not suffer from the previous injury played the forward position in lacrosse. This means that the twin spent most of their time running in short spaces during a game. This is a fast-paced style requiring lots of changing of direction and speed. However, the twin that had the previous non-contact ACL injury played the midfield position in lacrosse. This position requiredboth the rapid change of movement style of offensive play and the more linear style of play in the defensive position. Thus, the twin has to spend time training in ways with rapid, quick directional movement change and in the linear, crouched style of play from defense. Among the risks for an ACL injury, one of the more common risks is a quick change of direction with the foot firmly planted.

Just based upon the style of play between the twins, it is difficult to say which one was potentially at a higher risk for an ACL injury. Possibly, due to having to switch between two different styles of play in the game, both offensive style and defensive style, the twin who had the non-contact ACL injury was more at a risk. However, this cannot be confirmed unless more studies are done looking at the rate of non-contact and contact ACL injuries based upon position in lacrosse.

In terms of the return to sport criteria, even though there are more than one universal criteria, the twin with the previous injury had managed to fulfill a return to her sport. In addition, she also had a biomechanical profile that was on pace with the rest of the cohort. This is likely a testament to the extensive physical therapy and training she did to be able to return to play. In addition, it could also have been helped by her own personal training, like picking up the hobby of surfing. The purpose of this study was to further evaluate the return to sport criteria in two ways. One was to examine a return to sport in physical play after an ACL injury. The other was to directly look at the biomechanical profile in an athlete after an ACL injury and see if they had achieved a similar profile as individuals who were never injured. In terms of the identical twin who had the non-contact ACL injury, she had fulfilled both.

Due to there not being global criteria for return to sport, further research in the field of sports injuries needs to be continued. This is especially true for the ACL injury, where by trying to identify potential risks could potentially reduce the mental and physical stress, financial cost, and time spent in recovering from an injury. Furthermore, by continuing to research what is needed to fulfill successful rehabilitation, not just in the ability to play, but also in the individual's biomechanics, additional injuries can potentially be avoided. This is where research in the return to sport criteria is most 
important, to hopefully help prevent re-injury and secondary injuries.

\section{Acknowledgments}

1. There are no sources of funding. There is a $\$ 2,500$ credit towards supplies or presentations by the Frank H. Netter School of Medicine at Quinnipiac University.

2. Dr. Lynn Copes, my original mentor, helped guide my thoughts towards finding a topic to study. Dr. Karen Myrick, my main mentor, who helped find this particular study and guided me in the process from start of the study to completion. Dr. Juan Garbalosa, who supplied the original data and helped with the IRB and editing of the paper. Dr. Richard Feinn who helped me run data analysis and perform statistics for my paper.

\section{Conflicts of Interest}

1. There are no conflicts of interest.

2. The results of this study do not constitute endorsement by ACSM.

3. The results of this study are presented clearly and honestly without any fabrication, falsification, or inappropriate data manipulation.

\section{References}

1. Bradley JP, Klimkiewicz JJ, Rytel MJ, Powell JW (2002) Anterior cruciate ligament injuries in the National Football League. Arthroscopy: The Journal of Arthroscopic \& Related Surgery 18: 502-509.

2. Griffin LY, Agel J, Albohm MJ, Arendt EA, Dick RW, et al. (2000) Noncontact anterior cruciate ligament injuries: risk factors and prevention strategies. J Am Acad Orthop Surg 8: 141-150.

3. Hawkins RD, Hulse MA, Wilkinson C, Hodson A, Gibson M (2001) The association football medical research programme: an audit of injuries in professional football. British Journal of Sports Medicine 35: 43-47.

4. Hawkins R, Fuller CW (1999) A prospective epidemiological study of injuries in four English professional football clubs. British Journal of Sports Medicine 33: 196-203.

5. Landry S, McKean K, Hubley-Kozey C, Stanish W, Deluzio K (2007) Neuromuscular and Lower Limb Biomechanical Differences Exist between Male and Female Elite Adolescent Soccer Players during an Unanticipated Run and Crosscut Maneuver. The American Journal of Sports Medicine 35: 1901-1911.

6. Chaudhari A, Hearn B, Andriacchi T (2005) SportDependent Variations in arm Position during Single-Limb Landing Influence Knee Loading. The American Journal of Sports Medicine 33: 824-830.

7. Shelbourne K, Gray T, Haro M (2009) Incidence of Subsequent Injury to Either Knee within 5 Years after Anterior Cruciate Ligament Reconstruction with Patellar
Tendon Auto graft. The American Journal of Sports Medicine 37: 246-251.

8. Pinczewski L, Lyman J, Salmon L, Russell V, Roe J, et al.(2007) A 10-year comparison of anterior cruciate ligament reconstructions with hamstring tendon and patellar tendon autograft: a controlled, prospective trial. Scandinavian Journal of Medicine \& Science in Sports 17: 611-611.

9. Alentorn-Geli E, Myer G, Silvers H, Samitier G, Romero D, et al. (2009) Prevention of non-contact anterior cruciate ligament injuries in soccer players. Part 1: Mechanisms of injury and underlying risk factors. Knee Surgery, Sports Traumatology, Arthroscopy 17: 705-729.

10. Hewett T, Myer G, Ford K, Heidt R, Colosimo A, et al. (2005) Biomechanical Measures of Neuromuscular Control and Valgus Loading of the Knee Predict Anterior Cruciate Ligament Injury Risk in Female Athletes: A Prospective Study. The American Journal of Sports Medicine 33: 492-501.

11. McLean SG, Neal RJ, Myers PT, Walters MR (1999) Knee joint kinematics during the sidestep cutting maneuver: potential for injury in women. Medicine \& Science in Sports \& Exercise 31: 959-968.

12. McLean SG, Huang $X$, van den Bogert A (2005) Association between lower extremity posture at contact and peak knee valgus moment during sidestepping: Implications for ACL injury. Clinical Biomechanics 8: 863-870.

13. Hashemi J, Mansouri H, Chandrashekar N, Slauterbeck JR, Hardy DM (2011) Age, sex, body anthropometry, and ACL size predict the structural properties of the human anterior cruciate ligament. Journal of Orthopaedic Research 29: 993-1001.

14. Chaudhari AM1, Lindenfeld TN, Andriacchi TP, Hewett TE, Riccobene J, et al. (2007) Knee and Hip Loading Patterns at Different Phases in the Menstrual Cycle. The American Journal of Sports Medicine 35: 793-800.

15. Barengo NC, Meneses-Echávez JF, Ramírez-Vélez R, Cohen DD, Tovar G, et al. (2014) The Impact of the FIFA $11+$ Training Program on Injury Prevention in Football Players: A Systematic Review. International Journal of Environmental Research and Public Health 11: 1198612000.

16. Rössler R, Donath L, Bizzini M, Faude O (2015) A new injury prevention programme for children's football FIFA 11+ Kids - can improve motor performance: a cluster-randomised controlled trial. Journal of Sports Sciences 34: 549-556.

17. Silvers-Granelli H, Mandelbaum B, Adeniji O, Insler S, Bizzini M, et al. (2015) Efficacy of the FIFA 11+ Injury Prevention Program in the Collegiate Male Soccer Player. The American Journal of Sports Medicine 43: 2628-2637.

18. Pappas E, Nightingale E, Simic M, Ford K, Hewett T, et al. (2014) Do exercises used in injury prevention programmes modify cutting task biomechanics? A systematic review with meta-analysis. British Journal of Sports Medicine 49: 673-680.

19. Dragoo J, Braun H, Durham J, Chen M, Harris A (2012) Incidence and Risk Factors for Injuries to the Anterior 
Cruciate Ligament in National Collegiate Athletic Association Football. The American Journal of Sports Medicine 40: 990-995.

20. Agel J, Rockwood T, Klossner D (2016) Collegiate ACL Injury Rates Across 15 Sports. Clinical Journal of Sport Medicine 26: 518-523.
21. Barber-Westin S, Noyes F (2011) Factors Used to Determine Return to Unrestricted Sports Activities After Anterior Cruciate Ligament Reconstruction. Arthroscopy: The Journal of Arthroscopic \& Related Surgery. 27: 1697-1705.

Citation: Nowland M, Myrick K, Copes L, Garbalosa JC, Feinn R (2020) Comparison of Non-Contact ACL Injury vs No Injury in Female Identical Twin Athletes. Adv Ortho and Sprts Med: AOASM-120. 